\title{
CENTRALIZANDO INFORMAÇÕES DE PROCESSO E DE QUALIDADE UTILIZANDO O PIMS*
}

\section{Resumo}

\author{
Eduardo Batista Teixeira ${ }^{1}$ \\ Élcio Rodrigues Aranha ${ }^{2}$ \\ Eduardo do Carmo Silva ${ }^{3}$ \\ Guilherme Augusto Santiago Tavares ${ }^{4}$ \\ Pedro Henrique Moura Costa \\ Nathalie Martins Panoeiro 5
}

As indústrias podem possuir diversos sistemas especialistas para a gestão de cada uma das dimensões do negócio e, no caso, dos dados de análise a gestão de dados de laboratório a funcionalidade se dá com o LIMS, Sistema de gerenciamento de dados de laboratório (Laboratory Information Management System) e o Sistemas para o armazenamento de dados históricos de processo, PIMS, (Plant Information Management System). A correlação de dados de processo com dados de qualidade (resultados de análises de laboratório) é fator crítico de sucesso para o acompanhamento e gestão dos processos produtivos. Na intenção de atender as demandas dos usuários das informações correlacionadas, procuram guardar dentro do PIMS as informações do LIMS que agreguem valor para estes usuários. Cada variável historiada no PIMS consome uma posição de armazenamento (TAG). O quantitativo de TAG é parâmetro de licenciamento das soluções PIMS e o uso indiscriminado destes pode inviabilizar o projeto na dimensão custo. O presente trabalho apresenta solução para cadastramento dos resultados de análise utilizando estratégia de hierarquização no banco de dados. Esta estratégia reduzir para $20 \%$ (economia de $80 \%$ de TAG) do total de posições de armazenamento apuradas durante o levantamento de dados caso fosse utilizada a prática convencional de cadastramento dos dados. A hierarquização de dados reduz consideravelmente a demanda de TAG e não onera o processamento dos dados no momento da pesquisa e apresentação dos dados.

Palavras-chave: PIMS; LIMS; UFL; Integração de dados.

\section{Abstract}

\section{CENTRALIZING PROCESS AND QUALITY INFORMATION USING PIMS}

Industries can have multiple specialist systems to manage each dimension of the business. When talking about data analyses and management of laboratory data, the management specialist systems are the LIMS (Laboratory Information Management System) and the PIMS (Plant Information Management System) for process data storage. The correlation between process data and quality data (result of laboratory analyses) is a critical success factor for monitoring and management of production processes. Intending to attend the user's requests for correlated information, the LIMS data were stored inside PIMS thus adding value to these information. Each variable historiated in PIMS consumes a storage position (TAG). The number of TAG is the licensing parameter of PIMS solutions and its indiscriminate use may derail the project cost. The present work presents a solution for registration of analyze results using the database hierarchy strategy. This strategy can reduce to $20 \%$ (economy of $80 \%$ of TAG) of total storage positions established during the data survey when compared to conventional practice for data registration. The data hierarchy reduces significantly the TAG demand and doesn't burden the processing during search or presentation of data.

Keywords: PIMS; LIMS; UFL; Data integration.

\footnotetext{
Especialista, Analista, Docente e Pesquisador. Vale Fertilizantes, UNIP, Campus Santos, SP, Brasil Mestre, Engenheiro, Docente e Pesquisador. Vale Fertilizantes, UFABC, IFSP, Campus Cubatão, SP, Brasil. Gerente de Projeto, TSA Tecnologia de Sistemas de Automação, Belo Horizonte, MG, Brasil. Eng. de Automação, TSA Tecnologia de Sistemas de Automação, Belo Horizonte, MG, Brasil. Mestranda, Eng. de Automação, TSA Tecnologia de Sistemas de Automação, Belo Horizonte, MG, Brasil.
} 


\section{INTRODUÇÃO}

Os Sistemas de Gerenciamento de Informações de Processo, PIMS (Plant Information Management System) podem oferecer mais funcionalidades do que a aquisição de dados de diversos sistemas de informações e a simples armazenagem destes dados em um repositório único. É comum se deparar com a expressão "você não pode controlar o que você não pode ver" para exemplificar os benefícios de um sistema de gestão associado a métricas e um sistema de acompanhamento de modo geral. A deficiência no acesso e contextualização das informações pode acarretar aos gestores uma reação inadequada às demandas do negócio em seu trabalho diário. Os sistemas PIMS passaram a oferecer, ao longo dos anos, funcionalidades para a contextualização e hierarquia de dados em seu pacote básico de soluções. Esta integração e contextualização de dados podem ajudar os gestores na análise e tomada de decisão deixando o processo de gestão mais eficiente e com uma menor necessidade de entendimento de especificidades do sistema produtivo [7]. Com uma ferramenta padrão de consultas para este sistema, a visualização de forma única e normalizada dos dados históricos como os recémcoletados (ditos em base de tempo real) torna essa gestão rápida e eficiente. Estas informações podem ter as unidades de engenharia ajustadas e com grande velocidade de acesso. Estas ferramentas para o acesso à informação do PIMS permitem que tabelas, gráficos de tendências, telas sinópticas e relatórios dinâmicos possam ser disponibilizados a todos os interessados da corporação sem significativas necessidades de treinamento [2].

Porém, apesar dessa padronização das consultas, é sabido que as organizações possuem uma vasta quantidade de tipos de dados, obtidos das diversas fontes de informações da empresa, que podem ter diferentes definições e identificações tornando um complicador para análise dessas informações. A despadronização da natureza das informações armazenadas pode adicionar considerável complexidade até mesmo para as análises mais simples destes dados [4,9].

Dado a heterogeneidade da natureza dos dados em uma planta industrial, o sistema PIMS que está situado no centro da pirâmide conceitual de automação como mostra a Figura 1 - Pirâmide Conceitual de Automação, é um facilitador na integração entre sistemas de automação com sistemas de informação, com isso, está evoluindo cada vez mais para uma estrutura mais robusta e provida de soluções que permitem o historiamento dos dados distintos existentes em uma indústria. Um PIMS que conte com a hierarquia de dados bem definida pode beneficiar a implantação das inúmeras soluções que utilizam os dados históricos. Algumas das principais funcionalidades que pode-se citar como as mais comuns que utilizam dados de processo, a reconciliação de dados, o SCM (Suply Chain Manager), o MES (Manufactoring Execution System), a integração com sistemas como o ERP (Enterprise Resource Planning) e a implantação de sistemas especialistas, gerenciadores de desempenho e controles avançados [8].

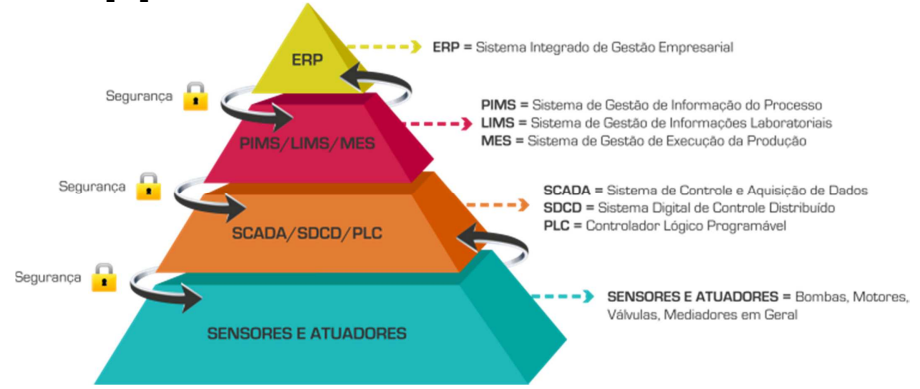

Figura 1. Pirâmide Conceitual da Automação. 
Apesar do exposto, sabe-se que grandes corporações, geralmente, possuem sistemas corporativos de informação, mas nem sempre estes sistemas podem ser integrados com facilidade com as informações do chão de fábrica e de qualidade do produto. Fornecedores de sistemas de automação afirmam que as indústrias de diversos segmentos, a partir da utilização mais abrangente das informações do processo, podem melhorar muito a sua lucratividade. Há indícios de significativas diferenças de lucratividade entre empresas que adotam a integração de seus sistemas com as empresas que ainda não o fizeram. Em alguns casos, a diferença de lucratividade, pode ser maximizada desde que a integração das informações dos sistemas de controle e gestão seja constituída e corretamente utilizada na tomada de decisão [5]. A hierarquização de dados pode ser um eficiente catalisador para a integração dos dados de processo com a camada corporativa da organização.

Funcionalidades para a contextualização e hierarquização de dados propiciariam, para os usuários do sistema, a possibilidade de encontrar rapidamente a informação necessária e utilizá-las de forma consistente para a tomada de decisões críticas $[1,6]$.

Este trabalho descreve uma proposta de atendimento de uma demanda de publicação de dados de análise de laboratório em um sistema PIMS utilizando a hierarquização de dados para minimizar o custo, o esforço de implantação e facilitar a análise dos dados históricos através da contextualização das informações. Para a publicação dos dados de laboratório no PIMS, foi provida uma solução simples e eficiente através de ferramentas nativas disponibilizadas pela ferramenta PIMS desenvolvida pela OSISoft [3]. Nesta integração foi utilizada uma interface de dados denominada PI UFL (Universal File Loader) que consulta arquivos em compatibilidade com o padrão ASCII, arquivos estes que são extraídos do sistema ERP da empresa [6]. O trabalho encontra-se então dividido da seguinte forma: na seção dois encontra-se um detalhamento da solução desenvolvida e na seção três encontram-se as conclusões, destacando os principais pontos e resultados da solução.

\section{CARACTERÍSTICAS E NECESSIDADES DO DESENVOLVIMENTO REALIZADO}

As necessidades para a gestão das empresas, visualização dos dados e seus relacionamentos podem evoluir e ter a forma de análise modificada ou acrescida de mais necessidades. Um sistema PIMS, para aumentar a sua eficiência, precisa disponibilizar ferramentas versáteis que possam atender as demandas de visualização e contextualização dos dados. Este trabalho não tem a pretensão de comparar e/ou avaliar o potencial das ferramentas concorrentes, mas sim descrever uma aplicação realizada que permite identificar os elementos que compõem um processo de publicação de resultados de análise de laboratório, especificar as relações entre os objetos e organizá-los de maneira que faça sentido para o seu negócio.

A solução apresentada foi desenvolvida a partir de duas necessidades:

Correlacionar de forma rápida, automatizada e eficiente dados de processo com dados de laboratório, em uma plataforma comum aos complexos industriais e ao mesmo tempo viabilizar a visualização das informações para grandes grupos de usuários com óticas distintas, entretanto, com interesses comuns no que se diz à melhoria contínua do processo produtivo.

Reduzir a utilização do uso de TAG's disponíveis no banco de dados, visto que utilizando-se métodos tradicionais de cadastramento de informações seria 
necessária a criação de mais de mil posições no banco de dados (considerando uma posição para informação/parâmetro das análises realizadas).

Para este trabalho, existia também a premissa de que se aproveitasse ao máximo os recursos tecnológicos já existentes na empresa, garantindo baixo investimento financeiro, mas com alta qualidade e confiabilidade da solução.

O projeto foi então aplicado em quatro complexos industriais participantes, sendo que todos usam o ERP para registro das informações de análises laboratoriais de maior relevância devido à ausência de um sistema LIMS, contudo, estas informações não contemplam todas as necessidades por parte dos usuários. Há de se considerar que o ERP não possui foco e consequentemente não contempla meios para integrar estes dados com dados de processo.

Além dos itens já mencionados é importante salientar que o número de usuários ERP também representa um custo significativo, dificultando 0 acesso às informações.

Dados os fatos apresentados, existem pontos relevantes que inviabilizam a solução para o problema baseado na plataforma ERP.

Alternativa seria a aquisição de um sistema LIMS, que além de fugir da premissa de utilizar tecnologias existentes, implicaria em dificuldades operacionais e técnicas para correlação das informações laboratoriais com as informações de processo. Além disso, existiria um ofensor quanto ao número de licenças necessárias para atender alta demanda de usuários, resultando em alto custo para a implantação e baixo retorno operacional/financeiro com esta aquisição.

\section{A SOLUÇÃO PIMS ADOTADA}

Como os dados de laboratório estão e permanecerão abrigados no ERP, este será o provedor das informações para o PIMS. Mas como fazer com que os dados cheguem ao PIMS, despendendo o menor custo sem abrir mão da confiabilidade?

Este tipo de integração normalmente é realizado através de softwares de interface (midlewares), desenvolvimento de drivers ou módulos conectores fornecidos pelos fabricantes das soluções. Neste caso, ao analisar ambas ferramentas a serem integradas, pôde ser notado uma transição já existente do lado do ERP, onde periodicamente é gerado um arquivo de texto contendo o detalhe das análises laboratoriais. A solução a ser implantada, até que o LIMS seja consolidado nos complexos, é a utilização de uma interface específica do PIMS para a leitura de arquivos em compatibilidade com o padrão ASCII.

Assim acrescentou-se à rotina de escrita do ERP, a emissão de arquivo texto em um diretório comum aos dois sistemas para que a interface de coleta do PIMS possa consumir tal arquivo, enviando as informações desejadas ao banco de dados do PIMS estabelecendo assim a integração unidirecional desses sistemas.

Porém, além da integração do PIMS com dados laboratoriais abrigados no ERP, tinha que ser considerado também o objetivo de otimizar a utilização dos registros (TAG's) disponíveis do banco de dados, onde sem a solução, a utilização de todas as posições no banco de dados inviabilizaria a integração e consequentemente o desenvolvimento da aplicação. Um estudo mais aprofundado nas funcionalidades disponíveis foi executado e o desempenho das alternativas encontradas para a hierarquização de dados foi considerado.

Levantou-se as características dos pontos de medição e cadastrados então os resultados no banco de dados. Foram criadas posições no banco de dados do tipo texto (uma para cada ponto de medição) para que as informações fossem 
armazenadas. Este agrupamento reduziu significamente a quantidade necessária de posições do banco de dados para menos de $50 \%$ do total previsto inicialmente (método tradicional de cadastramento e registro histórico).

Assim todos os resultados de um mesmo ponto de análise foram concatenados em uma única posição do tipo texto do banco de dados. Cada uma das informações é separada das outras pelo caractere "l". No momento da gravação da informação no banco de dados o sistema completa com o caractere "mantendo cada uma das informações a mesma quantidade de caracteres.

A concatenação das informações em uma única posição pode reduzir consideravelmente a necessidade de posições no banco de dados, mas a rotina para pesquisa de uma parcela da informação demandaria grande esforço e entendimento do usuário. Este fato inviabilizaria a utilização da informação por um usuário que não tenha pleno entendimento da estrutura do dado armazenado.

Após o problema apresentado, uma estrutura para estas variáveis foi definida e, a partir desta definição, o entendimento da organização da estrutura no banco de dados passou a ser transparente aos usuários. Eles passaram a acessar cada um dos resultados como se fosse uma variável isolada dispensando assim uma rotina complexa para a extração dos dados. Assim a declaração da hierarquia foi disponibilizada no sistema e os usuários passaram a acessar cada um dos resultados pelo respectivo atributo utilizado para a análise: $\mathrm{H} 2, \mathrm{~N} 2, \mathrm{CH} 4, \ldots \mathrm{O}$ usuário passa a pesquisar cada um dos resultados por uma nomenclatura simples e corriqueira: P1_H2 (ponto de coleta 1 - resultado de hidrogênio), P1_N2 (ponto de coleta 1 - resultado de nitrogênio) e assim sucessivamente. A Figura $\overline{2}$ - Detalhe da configuração no AF representa o descrito para a nomenclatura das posições do banco de dados histórico utilizando PI $A F \AA$ da OSIsof®.

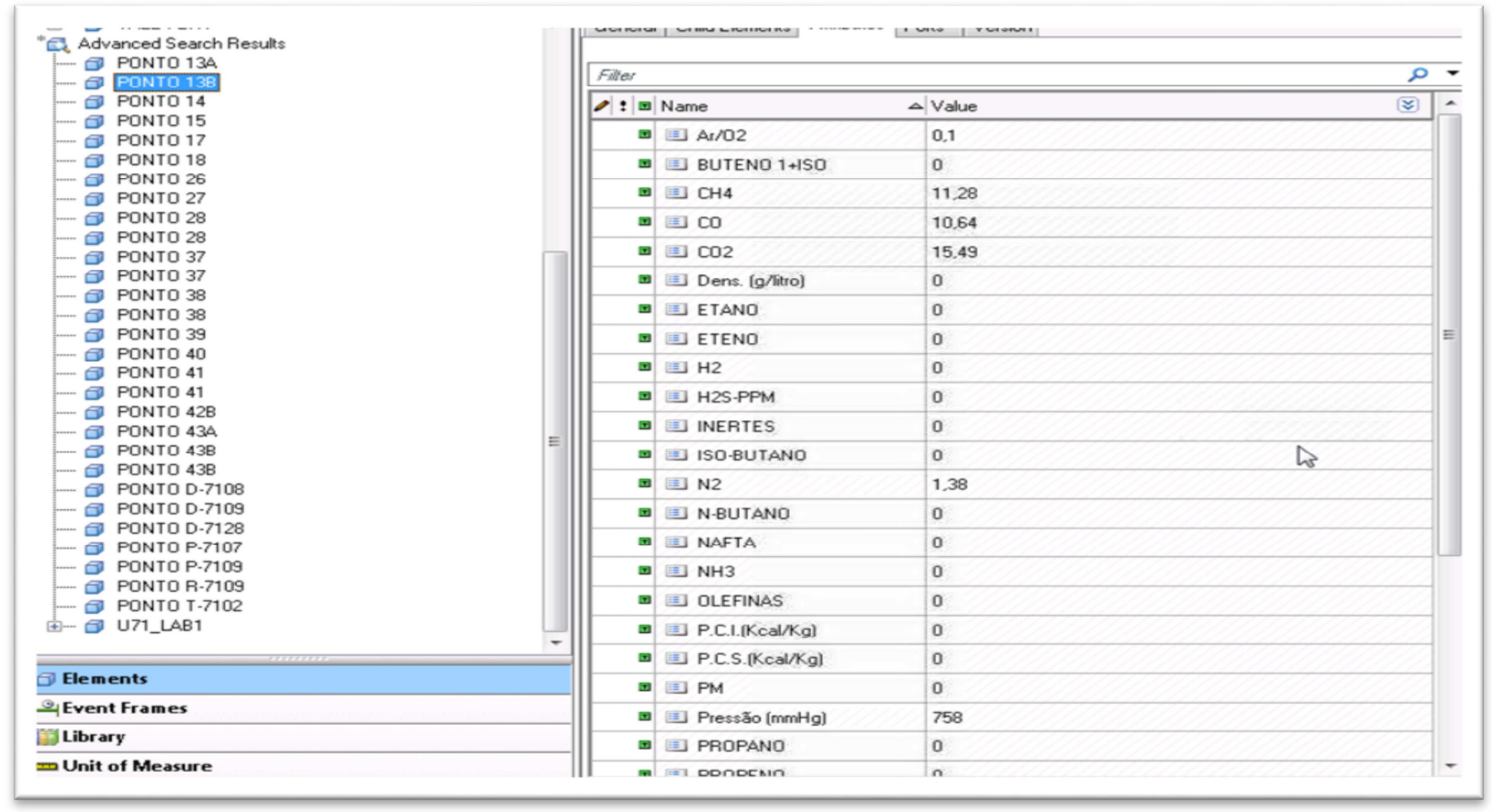

Figura 2. Detalhe da configuração no AF.

Ainda utilizando a ferramenta do OSIsoft® como exemplo, a Figura 3 - Detalhe de configuração, representa como os demais atributos receberam os valores usando como data reference a table lookup. 


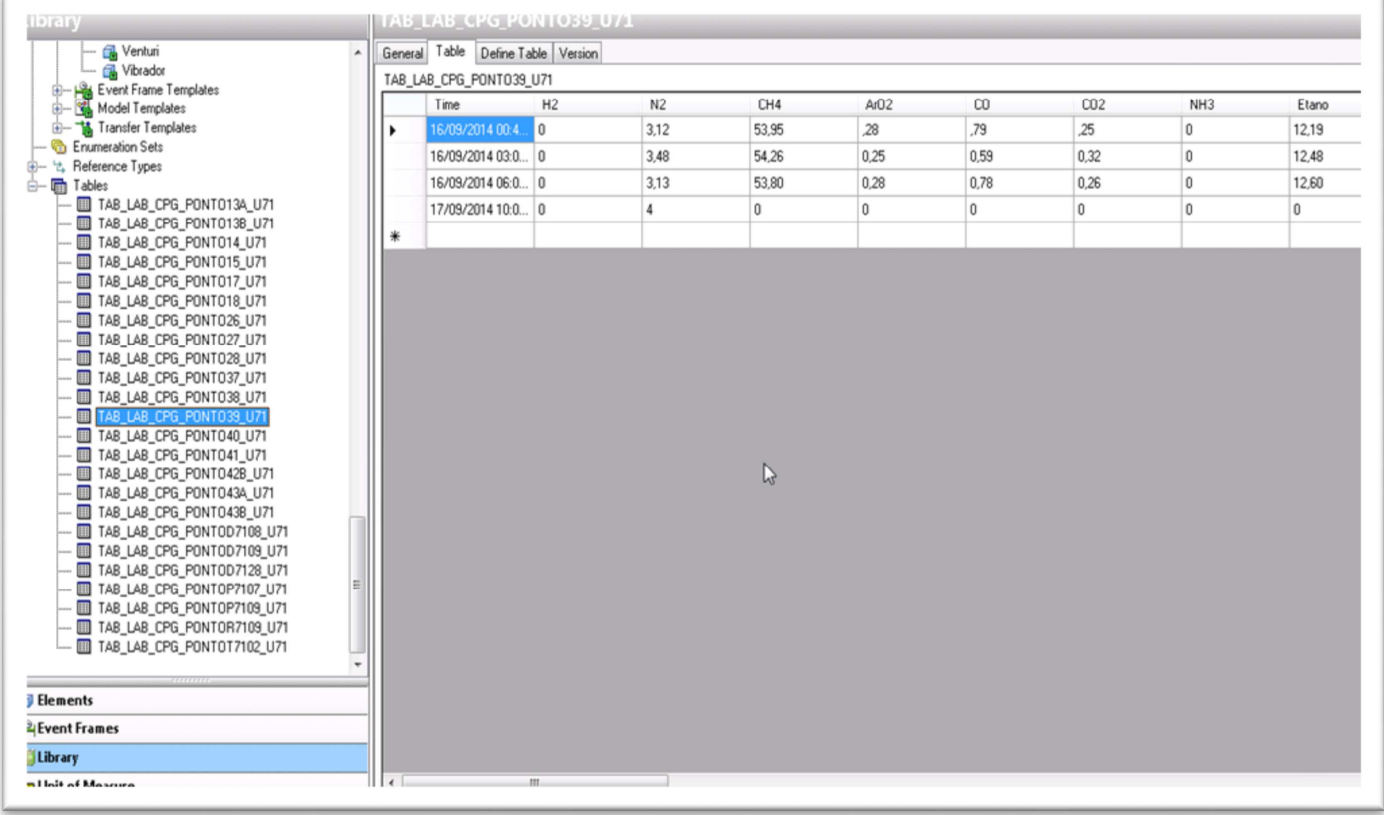

Figura 3. Detalhe da configuração (tabela no AF).

Cada atributo aponta para uma coluna específica da tabela do módulo de hierarquia. A tabela cria uma conexão "PIOLEDB Entreprise" com o banco relacional e realiza uma seleção (select) para o atributo da posição do banco de dados. Essa consulta traz para o usuário os valores históricos da porção específica solicitada da variável texto pesquisada.

Com esta hierarquia disponibilizada, qualquer aplicação cliente do PIMS pode utilizar as informações das análises em relatórios, gráficos de tendência, telas sinópticas, etc. de forma transparente (sem a necessidade de entendimento do formato utilizado para armazenamento). A Figura 4 - Exemplo de análise individual representa como a estrutura hierárquica pode ser utilizada de forma transparente pelo usuário.

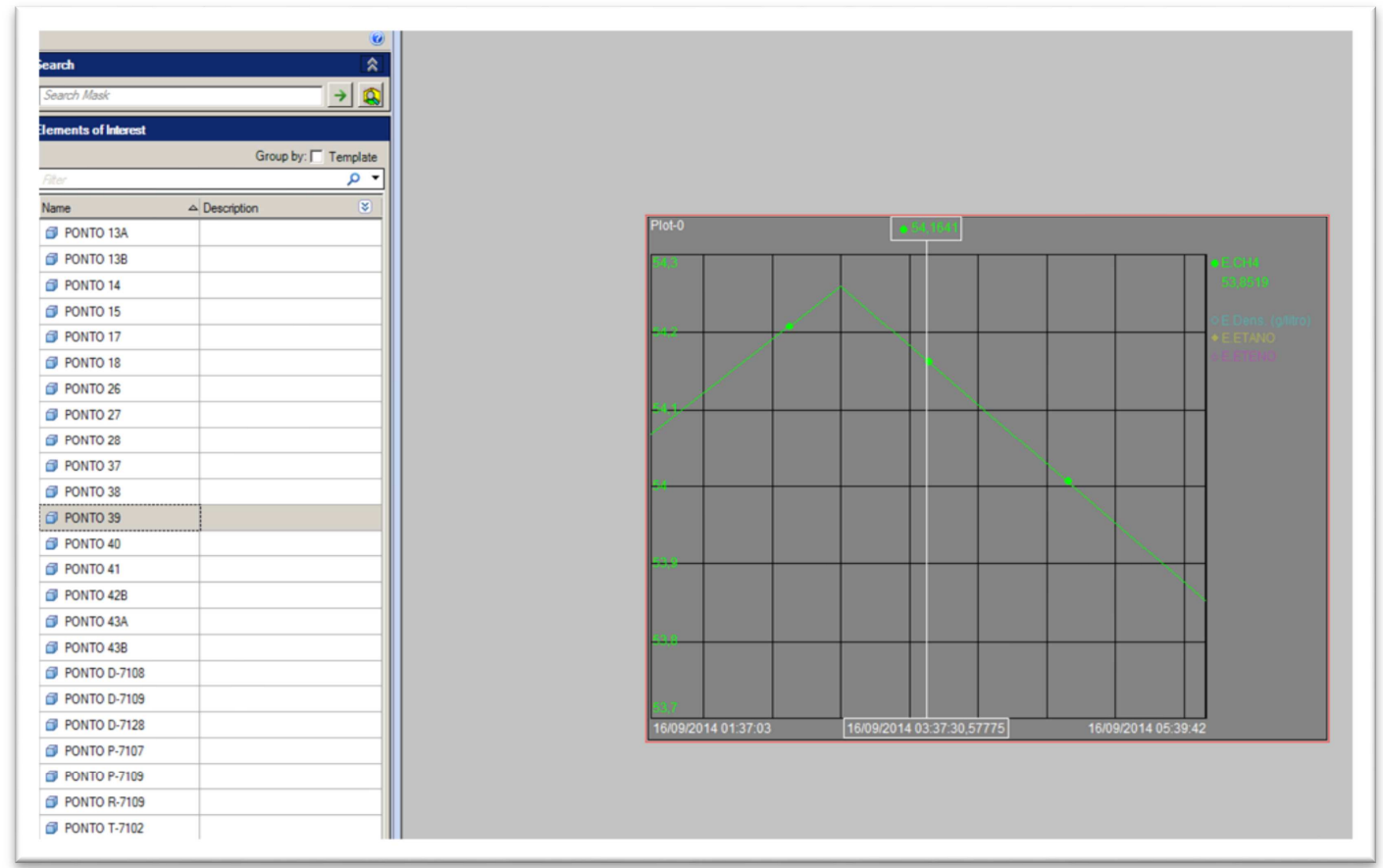

Figura 4. Exemplo de análise individual. 


\section{CONCLUSÃO}

Podem-se manter as bases operacionais existentes sem a alteração da filosofia do uso do ERP para registro das informações de maior relevância oriundas do laboratório e utiliza-se o PIMS que é comum aos quatro complexos para integrar com o primeiro. Essa solução atende às premissas de custo do projeto, possibilidade de acesso por um grande número de usuários e disposição de ferramentas analíticas. Além do exposto acima, a hierarquia de dados permite que as estruturas de informação disponíveis na camada operacional possam ser entendidas e utilizadas pelos sistemas da camada corporativa, sem que seja necessário um profundo conhecimento do sistema de índice das informações de processo na camada corporativa. Por fim, vale lembrar que por se tratar de uma solução nativa da ferramenta PIMS PI SYSTEM® torna-se simples sua implantação e reduz eventual número de intervenções por necessidade de configuração e manutenção. Somado aos itens já mencionados os usuários também são treinados no sistema PIMS o que propicia o uso familiar das múltiplas ferramentas clientes para a camada de visualização, tornando rápida e prática a consulta das informações bem como suas respectivas correlações.

\section{REFERÊNCIAS}

1 Aspentech - Aspen Manufacturing Master Data Manager - Getting Started Guide, Aspentech, Burlington - MA, 2014 [acesso em: 03 de out. de 2014]. Disponível em: <http://support.aspentech.com/webteamcgi/ValidateDownload.cgi?instructions $=5767421$ _Ghekjys3BBVcxW_MM7744AAAAQQ88521\&attachid=5937469\&docid=140765\&locati on=T>

2 Carvalho FB, Torres BS, Fonseca MO, Seixas C. Tecnologia em metalurgia e materiais. Sistemas PIMS - Conceituação, usos e benefícios. 2005;1:1-5.

3 Lobo J, Paredes R. Centro de monitoreo y Diagnostico, Xmo PI System Seminar, Santiago, agosto de 2007. Disponível em: www.osisoft.com (Events, Ucs and Webinars) acesso 30/09/2014.

4 Muller, C. Bascur, O. Campos, M. Gerenciamento de performance operacional em tempo real, Controle \& Instrumentação, Valete, n. 186, 2013.

5 Neves, J. M. S., Santos, F. C. A. Implantação de tecnologias de informação utilizadas na integração entre o chão-de fábrica e os sistemas ERP, Controle \& Instrumentação, Valete, n. 143, 2008.

6 OSISOFT - PI Asset Framework, OSISOFT, San Leandro - CA, 2014. Disponível em: $<$ http://www.osisoft.com/softwaresupport/products/product_info/DS_PI_AF_LT_EN.aspx> acesso em: 03 de out. de 2014.

7 Santiago Jr, J. R. S.; Santiago, J. R. S. Capital Intelectual - O Grande Desafio das Organizações. Brasil, Novatec, 2007, p. 308. ISBN 9788575221297

8 Seixas, C. Pims - Process Information Management System - Uma introdução, Universidade Federal de Minas Gerais, 2002.

9 Simonacci, R., Taccolini, M. Transformando informação em conhecimento, Controle \& Instrumentação, Valete, n. 186, 2013. 